\title{
Improved Cuckoo Search Algorithm for Nonconvex Hydrothermal Scheduling with Volume Constraint
}

\author{
Thang Trung Nguyen ${ }^{1}$, Dieu Ngoc Vo ${ }^{1}$, Tomas Deveikis ${ }^{2}$, Arnolda Rozanskiene ${ }^{2}$ \\ ${ }^{l}$ Power System Optimization Research Group, Faculty of Electrical and Electronics Engineering, \\ Ton Duc Thang University, HCM City, Vietnam \\ ${ }^{2}$ Department of Electrical Power Systems, Kaunas University of Technology, \\ Studentu St. 48, LT-51367 Kaunas, Lithuania \\ tomas.deveikis@ktu.lt
}

\begin{abstract}
This paper presents an improved cuckoo search algorithm (ICSA) for solving the short-term hydrothermal scheduling (ST-HTS) problem. In the proposed ICSA, the initial eggs are first classified into two groups including top group and abandoned group and each egg in the two groups is then newly generated via Lévy flights. The two new egg groups will be integrated into only one group before ranking and evaluating to choose the best egg, which will be used to generate new eggs via the action of discovery of alien eggs in the host bird's nest. Moreover, the fraction of the alien eggs to be abandoned will be adaptive at each iteration instead of a fixed value during the search process like the conventional Cuckoo Search Algorithm (CSA). The performance of the proposed ICSA has been tested on two different systems and the obtained results have been compared to those from many methods including CSA, modified CSA and other methods in the literature in terms of solution quality. The result comparison has revealed that the proposed ICSA is very efficient for solving the ST-HTS with reservoir storage constraints.
\end{abstract}

Index Terms-Improved Cuckoo search algorithm, shortterm hydrothermal scheduling, reservoir volume constraint.

\section{INTRODUCTION}

The short-term hydrothermal scheduling (ST-HTS) problem considers the optimization horizon from one day to one week involving hour-by-hour generation planning of all generating units in the hydrothermal system. The objective of the ST-HTS problem is to minimize the total generation fuel cost of thermal units while satisfying all constraints from hydropower plants including hydraulic constraint such as water discharge limits, volume reservoir limits, continuity water and generation limits, and thermal plant constraints including generation limits [1].

Several deterministic and meta-heuristic algorithms have been successfully applied for solving the ST-HTS problem such as simulated annealing approach (SA) [2], gradient search techniques (GS) [1], evolutionary programming technique (EP) [3]-[8], genetic algorithm (GA) [9]-[10], and clonal selection (CS) [11]. Among the methods, the SA is considered the worst optimization algorithm since it still suffers well-known drawback of high execution time for

Manuscript received 9 April, 2016; accepted 12 February, 2017. convergence and low solution quality. However, SA as well as other meta-heuristic algorithms like EP [3], [4], improved EP [5]-[8] are more effective than deterministic methods like GS [1] and lambda based Genetic algorithm [10] because the deterministic ones can not deal with problem considering nonconvex objective and nonlinear constraints [11].

Yang and Deb in [12] have developed CSA inspired by the intelligent reproduction behavior of cuckoo birds. This method has several advantages over PSO and GA such as better solution quality and higher success rate. CSA is one of the most modern meta-heuristic algorithms based on randomization structure and has become popular in power system optimization fields in recent years such as economic load dispatch [13] and short-term hydrothermal scheduling [14]. Another problem of control design in robotic filed considering several typical constraints including the limitations on positions, maximal velocities and servo-valve positions has been successfully performed by using GA, several PSO variants and CSA [15], and by GA, PSO, CSA and Bat algorithm (BA) [16]. The simulation results have indicated that CSA was superior to GA and PSO but less effective than BA in terms of capability of handling constraint conditions and optimal solutions. For the same problem with various types of proportional valves, and the combination of the cases of critical center, overlapped and underlapped valves, CSA has shown better efficiency than GA and PSO but also less performance than firefly algorithm (FA) [17]. In another study also about the optimal control of pneumatic-driven parallel robot platform [18], FA has also been successfully applied and resulted in promising improvement. The studies in [16], [17] have indicated that CSA has not been completely perfect for all optimization problems and there should be improvements in CSA. For a problem of chaotic systems parameter estimation, an improved CSA has been proposed by the combination of both the orthogonal design and simulated annealing operation [19]. The numerical results have proved that the algorithm could determine parameters with high accuracy and reliability, and its performance is better than CSA, GA and PSO. The study in [20] has pointed out that if the probability of alien eggs to be abandoned $P_{a}$ is small and the 
step size $\alpha$ in Lévy flights is large, CSA can converge to an optimal solution slowly. On the contrary, if the probability is large and the step size is small, CSA can get premature convergence without the best solution. As a result, the probability $P_{a}$ and the step size $\alpha$ have been modified in the study. $P_{a}$ and $\alpha$ have been considered as functions of the current iteration and their values have been changed during the search process where $P_{a}$ was increased but $\alpha$ was decreased. The high performance of the improved CSA has been validated by testing on four constrained problems related to design and by comparing with other methods and CSA. In [21], Walton et al. have pointed out that CSA has also owned a disadvantage in local search process entirely based on random walk. They have suggested two modifications on CSA in which the first modification is to consider the step size $\alpha$ in Lévy flights as a variable with the largest value at the beginning and the lowest value at the end while the second modification is the information exchange between two solutions for obtaining a new solution.The modified CSA (MCSA) has been demonstrated more effective and robust than CSA when applied to benchmark optimization functions [21] and short-term hydrothermal scheduling [22].

In this paper, an improved cuckoo search algorithm (ICSA) is proposed for solving the ST-HTS problem. The proposed ICSA is based on two modifications from MCSA method which was developed by Walton et al. [21]. In the ICSA, the initial eggs are first evaluated and classified into two groups including the good quality group and bad quality group. Each egg in the two groups is newly generated via Lévy flights. After that all new eggs are put in the integrated group and evaluated to determine the best egg corresponding to the lowest fitness function value. The best egg is used to generate new eggs in the second time via the action of alien egg discovery of the host bird. In addition, the probability of alien egg discovery in the proposed ICSA is adaptive at each iteration in stead of a fixed value as in CSA and MCSA. The performance of the proposed ICSA method has been tested on two systems with nonconvex fuel cost function of thermal units and power losses of transmission lines. The results obtained by the ICSA have been compared to those from several methods, CSA and MCSA.

\section{PROBLEM ForMULATION}

In this section, the mathematical formulation of the shortterm HTS problem consisting of $N_{l}$ thermal units and $N_{2}$ hydro units scheduled in $M$ time sub-intervals with $t_{m}$ hours for each is formulated. The objective function of the problem is as follows [22]

$$
F=\sum_{m=1}^{M} \sum_{i=1}^{N_{1}} t_{m}\left[\begin{array}{l}
a_{s i}+b_{s i} P_{s i, m}+c_{s i} P_{s i, m}^{2}+ \\
+\left|d_{s i} \times \sin \left(e_{s i} \times\left(P_{s i}^{\min }-P_{s i, m}\right)\right)\right|
\end{array}\right],
$$

where $a_{s i}, b_{s i}, c_{s i}, d_{s i}$, and $e_{s i}$ are fuel cost coefficients of thermal plant $i$.

Subject to:

1. Power balance constraint

$$
\sum_{i=1}^{N_{1}} P_{s i, m}+\sum_{j=1}^{N_{2}} P_{h j, m}-P_{L, m}-P_{D, m}=0,
$$

where $P_{D, m}$ and $P_{L, m}$ are total system load demand and power loss in the transmission line at subinterval $m ; P_{s i, m}$ and $P_{h j, m}$ are generations of thermal unit $i$ and hydro unit $j$ at subinterval $m$.

2. The total water discharge constraint: The available total water for all hydro units is determined by

$$
Q_{j, m}=t_{m} q_{j, m}
$$

where $q_{j, m}$ is water discharge rate obtained by

$$
q_{j, m}=a_{h j}+b_{h j} P_{h j, m}+c_{h j} P_{h j, m}^{2} .
$$

3. The continuity of reservoir volume: The water continuity of hydro power plants is represented by

$$
V_{j, m-1}-V_{j, m}+I_{j, m}-Q_{j, m}=0,
$$

where $V_{j, m}$ and $I_{j, m}$ are reservoir volume and water inflow of $j^{\text {th }}$ hydropower plant in $m^{\text {th }}$ interval, respectively.

4. Initial and final reservoir storage: The initial and final reservoir volumes are predetermined:

$$
\left\{\begin{array}{l}
V_{j, 0}=V_{j, \text { initial }}, \\
V_{j, M}=V_{j, \text { End }},
\end{array}\right.
$$

\section{Reservoir storage and Water discharge limits:}

$$
\begin{gathered}
V_{j, \text { min }} \leq V_{j, m} \leq V_{j, \max } ; j=1,2, \ldots, N_{2} ; m=1,2, \ldots, M, \\
q_{j, \min } \leq q_{j, m} \leq q_{j, \max } ; j=1,2, \ldots, N_{2} ; m=1,2, \ldots, M,
\end{gathered}
$$

where $V_{j, \max }$ and $V_{j, \min }$ are the maximum and minimum reservoir storage of the hydro plant $j ; q_{j, \max }$ and $q_{j, \min }$ are the maximum and minimum water discharge of the hydro plant $j$.

6. Generator operating limits:

$P_{s i, \min } \leq P_{s i, m} \leq P_{s i, \max } ; i=1,2, \ldots, N_{1} ; m=1,2, \ldots, M$,

$P_{h j, \min } \leq P_{h j, m} \leq P_{h j, \max } ; j=1,2, \ldots, N_{2} ; m=1,2, \ldots, M$,

where $P_{s i, \max }, P_{s i, \min }$ and $P_{h j, \max }, P_{h j, \min }$ are maximum, minimum power output of thermal plant $i$ and hydro plant $j$.

\section{Cuckoo Search Algorithm Based Methods for ST-HTS PROBLEM}

\section{A. Conventional Cuckoo Search Algorithm (CSA)}

CSA developed by Yang and Deb in 2009 [12] has been successfully applied for solving the short-term hydrothermal scheduling where reservoir volume constraint have been taken into account [14]. CSA has been considered more efficient than many methods including GS, EP variant, SA and other methods. 


\section{B. Modified Cuckoo Search Algorithm (MCSA)}

By performing modifications on CSA, Walton et al. have proposed MCSA to enhance the search ability of CSA. MCSA has been tested on benchmark functions and the results obtained by MCSA are better than those from CSA [19]. Moreover, MCSA is also more efficient than CSA for a hydrothermal scheduling problem [21]. In addition to three control parameters in CSA, MCSA also includes another parameter including the ratio of the number of nests in the top group to that in the abandoned group. The best value for this ratio was proposed to be 1:3 according to the study in [19]. Therefore, this ratio is also fixed at this value for the test cases in this paper.

\section{Improved Cuckoo Search Algorithm (ICSA)}

The proposed ICSA has been developed by combining MCSA [19] with modifications on the second new solution generation via discovery of alien eggs.

1. The first modification is to suggest an adaptive probability of eggs to reduce the number of independent trials corresponding to the probability. The adaptive probability is expressed in equation (11)

$$
P_{a}=P_{a, \max }-G \times\left(P_{a, \max }-P_{a, \min }\right) / G_{\max },
$$

where $G$ and $G_{\max }$ are the current iteration and the maximum iteration, respectively; $P_{a, \max }$ and $P_{a, \min }$ are the maximum and minimum probability of eggs to be abandoned.

To obtain the best solution by CSA and MCSA [19], the probability $P_{a}$ is set to a range of from 0.1 to 0.9 with a step of 0.1 . Thus, there are 9 different values of the probability need to be run. Instead, the proposed ICSA is performed with only three cases of $P_{a, \max }$ and $P_{a, \min }$ including $(0.9 ; 0.5)$, $(9.0 ; 0.1)$ and $(0.5 ; 0.1)$.

2. The second modification is to determine an increased value to update the new solution via the discovery of alien eggs. All the eggs in the top and abandoned groups after fulfilling the first new solution generation via Lévy flights are put in the integrated group and evaluated to choose the best one. The obtained best egg will be employed to obtain the increased value.

\section{IMPLEMENTATION OF THE PROPOSED IMPROVED CUCKOO SEARCH ALGORITHM FOR ST-HTS PROBLEM}

The proposed ICSA is a family of meta-heuristic algorithms. Thus, the application of the method for an optimization problem is also similar to other meta-heuristic algorithms like PSO, GA, and DE, etc. as long as given problems can be mathematically formulated as optimization problems consisting of objective function and constraints, which can be handled. For the considered ST-HTS problem, the main difficulty is to handle all constraints especially hydraulic constraints in reservoirs. The key of the high success rate is the selection of control variables and the selection of the slack variables. In the study, we have selected thermal units and reservoir volume as control variables while the first thermal unit and water discharges have been regarded as slack variables. The implementation of the proposed ICSA for solving the problem is described in detail as follows.

\section{A. Initialization}

The proposed ICSA has $N_{p}$ host nests, in which each host nest $X_{d}=\left[P_{s i, m, d}, V_{j, m, d}\right]$, where $P_{s i, m, d}$ and $V_{j, m, d}$ are power output of the $i^{\text {th }}$ thermal unit and reservoir volume of the $j^{\text {th }}$ hydropower plant at the $m^{\text {th }}$ subinterval corresponding to the $d^{\text {th }}$ nest among the fixed nests, respectively. $X_{d}$ is first randomly generated in the initialization within their limits.

The total water discharge over the $t_{m}$ hours is calculated using (5) as follows

$$
Q_{j, m}=V_{j, m-1}-V_{j, m}+I_{j, m}-S_{j, m} ; m=1, \ldots, M,
$$

The water discharge $q_{j, m}$ is calculated using (3) and then hydro generation $P_{h j, m}$ is obtained using (4) [14].

Thermal unit 1 is then obtained by [14]

$$
P_{s 1, m}=P_{D, m}+P_{L, m}-\sum_{i=2}^{N_{1}} P_{s i, m}-\sum_{j=1}^{N_{2}} P_{h j, m} .
$$

Based on the initial population of nests, the fitness function to be minimized corresponding to each nest is calculated by

$$
\begin{gathered}
F T_{d}=\sum_{m=1}^{M} \sum_{i=1}^{N 1} F_{i}\left(P_{s i, m, d}\right)+K_{s} \sum_{m=1}^{M}\left(P_{s 1, m, d}-P_{s 1}^{\lim }\right)^{2}+ \\
+K_{q} \sum_{j=1}^{N 2} \sum_{m=1}^{M}\left(q_{j, m, d}-q_{j}^{\lim }\right)^{2},
\end{gathered}
$$

where $K_{s}$ and $K_{q}$ are penalty factors for the slack thermal unit and water discharge, respectively; $P_{s l, m, d}$ is the generation of the slack thermal unit 1 at the $m^{\text {th }}$ subinterval of the $d^{\text {th }}$ nest calculated using (13).

The slack thermal unit limits and water discharge limits in (14) are determined as follows:

$$
\begin{aligned}
& P_{s 1}^{\lim }= \begin{cases}P_{s 1, \mathrm{max}}, & \text { if } P_{s 1, m, d}>P_{s 1, \mathrm{max}}, \\
P_{s 1, \mathrm{~min},}, & \text { if } P_{s 1, m, d}<P_{s 1, \mathrm{~min},} \quad m=1, \ldots, M, \\
P_{s 1, m, d}, & \text { otherwise, }\end{cases} \\
& q_{j}^{\lim }=\left\{\begin{array}{lll}
q_{j, \max }, & \text { if } q_{j, m, d}>q_{j, \max ,} & \\
q_{j, \min ,} \text { if } q_{j, m, d}<q_{j, \min ,} & j=1, . ., N_{2}, \\
q_{j, m, d}, & \text { otherwise, } & m=1, . ., M,
\end{array}\right.
\end{aligned}
$$

where $P_{s l, \max }$ and $P_{s l, \min }$ are respectively the upper and lower generations of the slack thermal unit.

Each nest is initially set to Xbest $_{d}\left(d=1, \ldots, N_{p}\right)$ and the nest whose the lowest fitness function value is set to the best nest Gbest.

\section{B. The First New Solution Generation via Lévy Flights}

As mentioned above, all nests in ICSA are first sorted in the descending order based on their fitness function value and then classified into two groups. The nests with high fitness function value Xabandoned $_{d}$ are put in abandoned group and the other ones $X$ top $_{d}$ are put in a top group. A nest 
which is randomly picked among the $X_{t o p}$ nests is called $X t_{\text {top }}$. Besides, another one with the best quality is named Xbest $_{d}$. The two new solution generations are respectively obtained as below.

1) Generation of new solution for the abandoned group

Based on the modification applied to the abandoned eggs with $d=$ Notop $+1, \ldots, N_{p}$ where Notop and $N_{p}$ are respectively the number of eggs in the top group and in the initial population, the optimal path for the Lévy flights is calculated using Mantegna's algorithm as follows

$$
\text { Xabandoned }_{d}^{\text {new }}=\left(\text { Xabandoned }_{d}+\alpha \times \text { rand } \times \Delta X_{d}\right),
$$

where rand is the distributed random number in $[0,1]$; the step size $\alpha$ is equal to $1 / \sqrt{G}$; and $\Delta X_{d}$ is obtained by

$$
\Delta X_{d}=v \times \sigma_{x}(\beta) \times\left(\text { Xabandoned }_{d}-\text { Xbest }_{d}\right) / \sigma_{y}(\beta) .
$$

\section{2) Generation of new solution for the top egg group}

The modification is applied to the eggs in the top group with $d=1, \ldots$, Notop. The optimal path for the Lévy flights is calculated using Mantegna's algorithm as follows

$$
X t o p_{d}^{n e w}=X t o p_{d}+\alpha \times \text { rand } \times \Delta X_{d} .
$$

The value of $\alpha$ and $\Delta X_{d}$ will be determined depending on considered cases as follows:

a) Case 1: The same egg is picked twice

$$
\Delta X_{d}=v \times \sigma_{x}(\beta) \times\left(X_{t o p}-X b e s t\right) / \sigma_{y}(\beta) .
$$

b) Case 2: Both eggs have the same fitness value function

$$
\Delta X_{d}=\left(X t o p_{d}-X t o p_{r}\right) / 2 .
$$

c) Case 3: The random egg has lower fitness than egg $d$

$$
\Delta X_{d}=\left(X t o p_{r}-X t o p_{d}\right) / \varphi
$$

or the random egg has higher fitness than egg $d$

$$
\Delta X_{d}=\left(X t o p_{d}-X t o p_{r}\right) / \varphi
$$

where $\alpha=1 / G^{2}$ for case 1 and $\alpha=1$ for cases 2,3 , and $\varphi=(1+\sqrt{5}) / 2$.

There are no criteria to ensure that the new solutions always satisfy their bounds. Therefore, they need to be defined as below:

$$
\begin{aligned}
& V_{j, m, d}=\left\{\begin{array}{lll}
V_{j, \max }, & \text { if } V_{j, m, d}>V_{j, \max }, & j=1, \ldots, N_{2}, \\
V_{j, \min }, & \text { if } V_{j, m, d}<V_{j, \min }, & m=1, \ldots, M-1,(24) \\
V_{j, m, d}, & \text { otherwise, }
\end{array}\right. \\
& P_{s i, m, d}=\left\{\begin{array}{lll}
P_{s i, \max }, & \text { if } P_{s i, m, d}>P_{s i, \max }, & i=2, \ldots, N_{1} \\
P_{s i, \min }, & \text { if } P_{s i, m, d}<P_{s i, \min }, & m=1, \ldots, M . \\
P_{s i, m, d,}, & \text { otherwise. }
\end{array}\right.
\end{aligned}
$$

The power output of $N_{2}$ hydro units and the slack thermal unit are then obtained by using (4) and (13), respectively. The fitness value is calculated using (14). The nest corresponding to the best fitness function is then set to the best nest Gbest.

\section{The Second New Solution Generation via Discovery of Alien Eggs}

Similar to MCSA, all eggs of the top group and abandoned group in the proposed ICSA are first integrated into one group and a fraction of all the eggs to be abandoned are newly generated via the action of alien egg discovery. However, in ICSA the probability of the eggs to be abandoned is adaptive at each iteration by using (11).

The new solution due to this action is found as follows

$$
X_{d}^{d i s}=X_{\text {best }}+K \times \Delta X_{d}^{d i s},
$$

where $K$ is the updated coefficient [13], [14], [23] and the increased value $\Delta X_{d}^{\text {dis }}$ is determined as below

$$
\Delta X_{d}^{d i s}=\operatorname{rand} \times\left[\operatorname{randp}\left(\text { Xbest }_{d}\right)-\text { Gbest }\right],
$$

where randp $\left(\right.$ Xbest $\left._{d}\right)$ is the random perturbation for position of the nests in Xbest $_{d}$.

\section{Terminating Criteria}

The proposed ICSA is stopped when the current iteration reaches the maximum number of iterations.

\section{E. Overall Procedure}

The overall procedure of the proposed ICSA for solving the ST-HTS problem is described as follows.

Step 1: Select parameters for the ICSA including $N_{p}$, $P_{a, \max }$ and $P_{a, \min }$, and $G_{\max }$.

Step 2: Initialize $N_{p}$ host nests as in Section IV.A and calculate the power output of all hydro units and the slack thermal unit using (4) and (13). Evaluate the fitness function using (14) to choose the best solution Gbest. Set the initial iteration counter $G=1$.

Step 3: Classify all eggs into two groups including top group an abandoned group.

Step 4: Generate new solutions via Lévy flights for abandoned group as Section IV.B.1.

Step 5: Generate new solutions via Lévy flights for top group as described in Section IV.B.2.

Step 6: Put the new eggs in top group and abandoned group into the integrated group and calculate the power output of all hydro units and the slack thermal unit using (4) and (13). Evaluate all the eggs to determine the best egg with the lowest fitness function, Gbest.

Step 7: Generate new solutions via the discovery of alien eggs as Section IV.C and calculate the power output of all hydro units and the slack thermal unit using (4) and (13).

Step 8: Evaluate the fitness function using (14) to determine the best egg, Gbest.

Step 9: If $G<G_{\max }, G=G+1$ and go back to Step 3. Otherwise, terminate the iterative procedure.

\section{Numerical RESUlts}

The electricity generation cost of each generating unit is a 
function of power output and other coefficients, which have been obtained by experiment. During the operation of the generating units, the valve point loading effects can be considered or neglected and the cost function for each case is different. For the case of neglecting the valve effects, the cost function is approximately represented as a second order equation called convex function but for the case of considering the valve effects, the cost function is approximately represented a nonconvex function, which is a sum of a convex function and a sinusoidal function similar to (1). The first function with convex form is easy for applying all methods while the second function with nonconvex form is a big challenge for deterministic methods. Consequently, in the study the proposed ICSA is verified on two systems with different forms of cost function in which the first one has one thermal plant with convex function and one hydro plant [1] and the other one consists of four thermal plants with nonconvex function and four hydro plants [14]. The two systems are scheduled in 3 days with six 12-hour intervals. The transmission losses are neglected in the first system but included in the second one. Both CSA and MCSA respectively described in Sections III.A and III.B are also implemented for solving the two systems for result comparison. The three algorithms of CSA, MCSA and ICSA are coded in Matlab platform with run fifty independent trials for each case on a $1.8 \mathrm{GHz}$ PC with $4 \mathrm{~GB}$ of RAM.

\section{A. System with Quadratic Fuel Cost Function of Thermal Units}

For this system, $N_{p}$ and $G_{\max }$ for of CSA, MCSA and ICSA are respectively set to 10 and 100 . The probability $P_{a}$ of CSA and MCSA is set to the range from 0.1 to 0.9 with a step of 0.1 and the value of $P_{a, \max }$ and $P_{a, \min }$ of ICSA are respectively set to 0.9 and $0.5,0.5$ and 0.1 , and 0.9 and 0.1 . Finally, the best results obtained are respectively at $P_{a}=0.6$ by CSA, $P_{a}=0.8$ by MCSA and $P_{a, \max }=0.9$ and $P_{a, \min }=0.5$ by ICSA. The summary of the results is given in Table I.

TABLE I. RESULTS OBTAINED FOR SYSTEM I.

\begin{tabular}{|c|c|c|c|}
\hline Method & CSA & MCSA & ICSA \\
\hline Best cost (\$) & 709862.05 & 709862.05 & 709862.05 \\
\hline Mean cost (\$) & 710016.55 & 709862.72 & 709862.13 \\
\hline Worst cost(\$) & 715532.97 & 709866.86 & 709862.83 \\
\hline Std. dev. (\$) & 809.643 & 1.262 & 0.16 \\
\hline CPU time (s) & 0.08 & 0.13 & 0.12 \\
\hline
\end{tabular}

The best cost from the three methods is compared to the best cost obtained by other methods as given in Table II.

TABLE II. COMPARISON OF RESULT OBTAINED FROM THE THREE CSA METHODS WITH OTHER METHODS FOR SYSTEM I.

\begin{tabular}{|c|c|c|c|c|c|}
\hline Method & Cost (\$) & $\begin{array}{c}\text { Time } \\
(\mathbf{s})\end{array}$ & Method & Cost (\$) & $\begin{array}{c}\text { Time } \\
(\mathbf{s})\end{array}$ \\
\hline GS [1] & 709877.38 & - & IFEP [5] & 709862.05 & 59.7 \\
\hline SA [2] & 709874.36 & 901 & RIFEP[6] & 709862.05 & - \\
\hline EP [3] & 709862.06 & 8 & CS [11] & 709862.05 & 4.54 \\
\hline EP [4] & 709863.29 & 264 & CSA & 709862.0494 & 0.08 \\
\hline CEP[5] & 709862.05 & 159.2 & MCSA & 709862.0491 & 0.13 \\
\hline FEP[5] & 709862.05 & 101.4 & ICSA & 709862.0490 & 0.12 \\
\hline
\end{tabular}

The comparison has revealed that the ICSA can obtain better solution than GS [1], SA [2], EP [3], and EP [4] and approximately good solution with that from other methods. Moreover, ICSA spends shorter simulation time than the most of methods excluding CSA for obtaining the best optimal solution. The convergence characteristics in Fig. 1 indicate that ICSA obtains better solution than CSA and MCSA at each iteration.

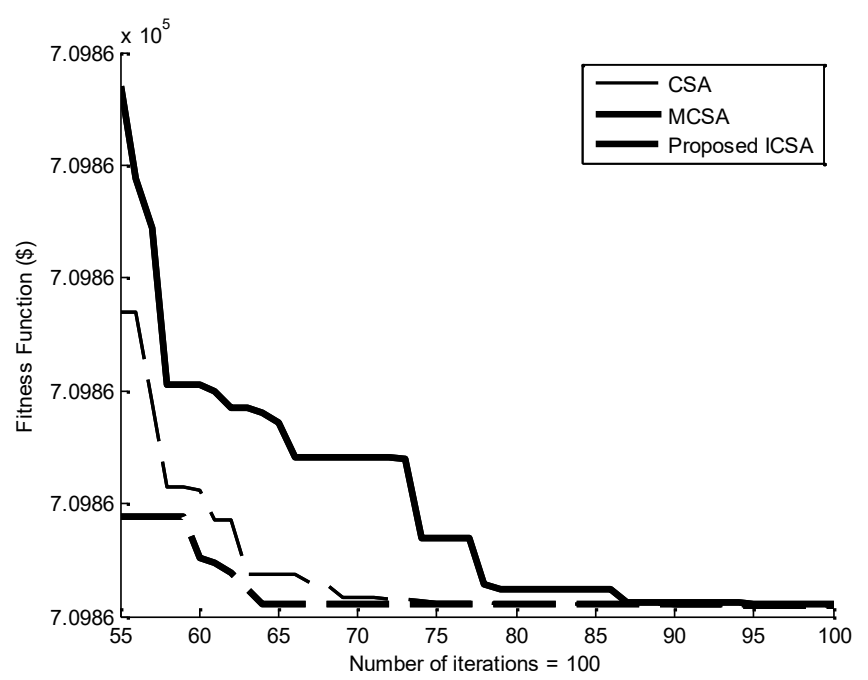

Fig 1. Convergence characteristics of the three methods for system I.

\section{B. System with Nonconvex Cost Function of Thermal Units}

For implementation of the methods, $N_{p}$ is set to 50 for CSA and 36 for both MCSA and the proposed ICSA whereas $G_{\max }$ is set to 3500 for all methods. Table III shows the result comparison among CSA methods. Obviously, the proposed ICSA can obtain better minimum, average and standard deviation cost than CSA-Cauchy, CSA-Gauss, CSA, and MCSA. On the other hand, the ICSA is faster than CSA, CSA-Cauchy, and CSA-Gauss. Therefore, ICSA is more efficient than both CSA-Cauchy, CSA-Gauss, CSA and MCSA for solving the large-scale hydrothermal system scheduling considering nonconvex fuel cost function of thermal units and power losses in transmission lines. Figure 2 indicates that the proposed method has better convergence characteristics than CSA and MCSA.

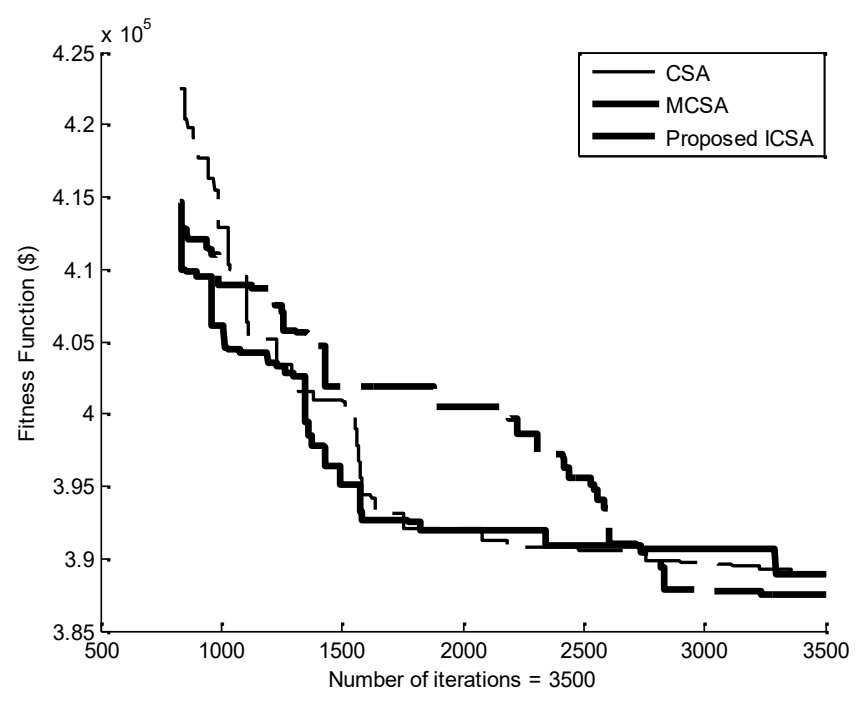

Fig. 2. Convergence characteristics of the three methods for system II. 
TABLE III. RESULTS OBTAINED FOR SYSTEM II.

\begin{tabular}{|c|c|c|c|c|c|}
\hline Method & $\begin{array}{c}\text { CSA- } \\
\text { Cauch } \\
\text { y [15] }\end{array}$ & $\begin{array}{c}\text { CSA- } \\
\text { Gauss } \\
{[\mathbf{1 5 ]}}\end{array}$ & CSA & MCSA & ICSA \\
\hline Best cost (\$) & 388888 & 389214 & 387726 & 388624 & 387512.6 \\
\hline Mean cost (\$) & 394450 & 400034 & 396693 & 395952 & 393059.2 \\
\hline Worst cost (\$) & 409168 & 495151 & 468641 & 406881 & 400526 \\
\hline Std. dev. (\$) & 4416 & 22239 & 11167 & 2622.5 & 2831.3 \\
\hline CPU time (s) & 49.9 & 48.3 & 47.6 & 35.4 & 34.6 \\
\hline
\end{tabular}

\section{CONCLUSIONS}

In this paper, three CSA methods including conventional CSA, modified CSA and the proposed improved CSA have been implemented for solving the short-term hydrothermal scheduling problem considering nonconvex fuel cost function of thermal units and power losses in transmission lines. Compared to CSA and MCSA, the proposed method can improve quality of the solutions obtained via the action of alien egg discovery and a suggested adaptive probability enables the proposed method to reduce a number of trials. The advantages of the proposed method have been tested on the two systems and the obtained results have indicated that the proposed ICSA method is more effective and robust than conventional CSA, modified CSA and many other methods. Therefore, the proposed ICSA is a very powerful metaheuristic algorithm for searching the optimal solution of the short-term hydrothermal scheduling problem with nonconvex objective function.

\section{REFERENCES}

[1] A. J. Wood, B. F. Wollenberg, Power Generation, Operation, and Control. New York: Wiley, 1996.

[2] K. P. Wong, Y. W. Wong, "Short-term hydrothermal scheduling, part-I: simulated annealing approach", IEE Proc. Part-C, vol. 141, 1994, pp. 497-501. [Online]. Available: http://dx.doi.org/10.1109/ TPWRS.2002.807053

[3] P. Yang, H. Yang, C. Huang, "Scheduling short-term hydrothermal generation using evolutionary programming techniques", IEE Proc. Generation Transmission and Distribution, 1996, pp. 371-376. [Online]. Available: http://dx.doi.org/10.1049/ip-gtd:19960463

[4] P. K. Hota, R. Chakrabarti, P. K. Chattopadhyay, "Short-term hydrothermal scheduling through evolutionary programming technique", Electric Power Systems Research, vol. 52, pp. 189-196, 1999. [Online]. Available: https://doi.org/10.1016/S03787796(99)00021-8

[5] N. Sinha, R. Chakrabarti, P. K. Chattopadhaya, "Fast evolutionary programming techniques for short-term hydrothermal scheduling", Electric Power Syst. Res., vol. 66, pp. 97-103, 2003. [Online]. Available: https://doi.org/10.1109/TPWRS.2002.807053

[6] B. Turkay, F. Mecitoglu, S. Baran, "Application of a fast evolutionary algorithm to short-term hydro-thermal generation scheduling", Energy Sources, Part B: Economics, Planning, and Policy, vol. 6, pp. 395-405, 2011. [Online]. Available: http://dx.doi.org/10.1080/15567249.2010.489098

[7] B. N. S. Rahimullah, T. K. A. Rahman, "Short-term hydrothermal generation scheduling using evolutionary computing technique", 4th Student Conf. Research and Development (SCOReD 2006), 2006, pp. 220-223. [Online]. Available: http://dx.doi.org/10.1109/ SCORED.2006.4339342

[8] N. Sinha, R. Chakrabarti, P. K. Chattopadhyay, "Fast evolutionary programming techniques for short-term hydrothermal scheduling", IEEE Trans. Power Systems, vol. 18, pp. 214-220, 2003. [Online]. Available: http://dx.doi.org/10.1109/TPWRS.2002.807053

[9] C. E. Zoumas, A. G. Bakirtzis, J. B. Theocharis, V. Petridis, "A genetic algorithm solution approach to the hydrothermal coordination problem", IEEE Trans. Power Systems, vol. 19, pp. 1356-1364, 2004. [Online]. Available: http://dx.doi.org/10.1109/TPWRS 2004.825896

[10] J. Sasikala, M. Ramaswamy, "Optimal gamma based fixed head hydrothermal scheduling using genetic algorithm", Expert Systems with Applications, vol. 37, pp. 3352-3357, 2010. [Online]. Available: https://doi.org/10.1016/j.eswa.2009.10.015

[11] R. K. Swain, A. K. Barisal, P. K. Hota, R. Chakrabarti, "Short-term hydrothermal scheduling using clonal selection algorithm", Electrical Power \& Energy Systems, vol. 33, pp. 647-656, 2011. [Online]. Available: https://doi.org/10.1016/j.ijepes.2010.11.016

[12] X. S. Yang, S. Deb, "Cuckoo search via Lévy flights", in Proc. World Congress on Nature \& Biologically Inspired Computing (NaBIC 2009), India, 2009, pp. 210-214. [Online]. Available: http://dx.doi.org/10.1109/NABIC.2009.5393690

[13] V. N. Dieu, S. Peter, W. Ongsakul, "Cuckoo search algorithm for non-convex economic dispatch", IET Generation, Transmission \& Distribution, vol. 7, pp. 645-54, 2013. [Online]. Available: http://dx.doi.org/10.1049/iet-gtd.2012.0142

[14] T. T. Nguyen, D. N. Vo, B. H. Dinh, "Cuckoo search algorithm using different distributions for short-term hydrothermal scheduling with reservoir volume constraint", Int. Journal on Electrical Engineering and Informatics, vol. 8, pp. 76-92, 2016. [Online]. Available: http://dx.doi.org/10.1109/TENCON.2014.7022454

[15] V. Stojanovic, N. Nedic, D. Prsic, L. Dubonjic, V. Djordjevic, "Application of cuckoo search algorithm to constrained control problem of a parallel robot platform", The International Journal of Advanced Manufacturing Technology, vol. 87, pp. 2497-2507, 2016. [Online]. Available: http://dx.doi.org/10.1007/s00170-016-8627-z

[16] V. Stojanovic, N. Nedic, "A nature inspired parameter tuning approach to cascade control for hydraulically driven parallel robot platform", Journal of Optimization Theory and Applications, vol. 168, pp. 332-347, 2016. [Online]. Available: http://dx.doi.org/ $10.1007 / \mathrm{s} 10957-015-0706-\mathrm{z}$

[17] N. Nedic, V. Stojanovic, V. Djordjevic, "Optimal control of hydraulically driven parallel robot platform based on firefly algorithm", Nonlinear Dynamics, vol. 82, pp. 1457-1473, 2015. [Online]. Available: http://dx.doi.org/10.1007/s11071-015-2252-5

[18] D. Prsic, N. Nedic, V. Stojanovic, "A nature inspired optimal control of pneumatic-driven parallel robot platform", in Proc. Institution of Mechanical Engineers, Part C: Journal of Mechanical Engineering Science, vol. 231, pp. 59-71, 2017. [Online]. Available: http://dx.doi.org/10.1177/0954406216662367

[19] J. Wang, B. Zhou, S. Zhou, "An improved cuckoo search optimization algorithm for the problem of chaotic systems parameter estimation", Computational intelligence and neuroscience, 2016 pp. 1-8. [Online]. Available: http://dx.doi.org/10.1155/2016 $/ 2959370$

[20] R. R Bulatovic, G. Boskovic, M. M. Savkovic, M. M. Gasic, "Improved cuckoo search (ICS) algorithm for constrained optimization problems", Latin American Journal of Solids and Structures, vol. 11, pp. 1349-1362, 2014. [Online]. Available: https://doi.org/10.1590/S1679-78252014000800004

[21] S. Walton, O. Hassan, K. Morgan, M. R. Brown, "Modified cuckoo search: A new gradient free optimisation algorithm", Chaos, Solutions \& Fractals, vol. 44, pp. 710-718, 2011. [Online]. Available: https://doi.org/10.1016/j.chaos.2011.06.004

[22] A. Dogan, T. Yalcinoz, M. Alci, "A comparison of heuristic methods for optimum power flow considering valve point effect", Elektronika ir Elektrotechnika, vol. 22, pp. 32-37, 2016. [Online]. Available: https://doi.org/10.5755/j01.eie.22.5.16340

[23] T. T. Nguyen, D. N. Vo, "Modified Cuckoo search algorithm for short-term hydrothermal scheduling", Electrical Power and Energy Systems, vol. 65, pp. 271-281, 2015. [Online]. Available: https://doi.org/10.1016/j.ijepes.2014.10.004 